\title{
Toroidal mode number determination of ELM associated phenomena on ASDEX Upgrade
}

\author{
Felician Mink ${ }^{1,2}$, Elisabeth Wolfrum ${ }^{1}$, Marc Maraschek ${ }^{1}$, \\ Hartmut Zohm ${ }^{1}$, László Horváth ${ }^{3}$, Florian M. Laggner ${ }^{4}$, Peter \\ Manz $^{2,1}$, Eleonora Viezzer ${ }^{1}$, Ulrich Stroth ${ }^{1,2}$, \\ the ASDEX Upgrade Team ${ }^{1}$ \\ ${ }^{1}$ Max-Planck-Institut für Plasmaphysik, Boltzmannstr. 2, 85748 Garching, Germany \\ ${ }^{2}$ Physik Department, E28, TUM, 85748 Garching, Germany \\ ${ }^{3}$ York Plasma Institute, University of York, Heslington, York, YO105DD, UK \\ ${ }^{4}$ Institute of Applied Physics, TU Wien, Fusion@ÖAW, 1040 Vienna, Austria \\ E-mail: felician.mink@ipp.mpg.de
}

August 2016

\begin{abstract}
In highly confined tokamak plasmas periodically appearing edge localized modes (ELMs) are accompanied by mode-like magnetohydrodynamic (MHD) activities with defined toroidal mode numbers.

Here the method of determining toroidal mode numbers $n$ on the ASDEX Upgrade tokamak with a toroidally spread magnetic pick-up coil array is reviewed and improved by taking into account intrinsic coil phases. ELM synchronization is used to characterize inter-ELM MHD activity and their development during the ELM cycle in terms of their mode numbers. The mode number development is correlated with the development of the pedestal parameters which shows that the inter-ELM modes cause transport across the pedestal. An estimation of the position of the modes is done via a comparison between the mode velocities and the plasma rotation profile at the edge.

Results show that during the ELM cycle MHD modes appear at several positions in the strong gradient region with clearly defined toroidal structures in the range of $n=1-10$. These structures of inter-ELM modes are preserved during the ELM crash where also a strong $n=0$ phenomenon occurs.
\end{abstract}

Keywords: Edge localized mode, magnetic pick-up coils, mode numbers, pedestal

\section{Introduction}

Edge localized modes (ELMs) occur as repetitive bursts of magnetohydrodynamic (MHD) activity in the high-confinement regime (H-mode) of tokamak fusion plasmas. According to the broadly accepted peeling-ballooning model these MHD instabilities are driven by the steep edge pressure and current gradients, which are characteristic for the 
H-mode $[1,2]$. Nevertheless, the process that determines the onset of an ELM crash is not completely understood. As ELMs lead to a sudden release of pedestal stored energy they might cause intolerably high heat fluxes onto the divertor target plates or the first wall in future fusion devices like ITER [3]. Therefore, it is necessary to gain a better understanding of the process that triggers the ELM.

It has been reported from different tokamaks that in the inter-ELM phase periodic MHD activities that might be connected to the ELM crash can be observed with defined toroidal mode numbers $n[4,5,6]$. As the toroidal mode number is a key parameter for the characterization of modes it is important to determine it for these inter-ELM phenomena to understand their connection to the ELM. Investigation of such mode structures in terms of $\mathrm{n}$ is usually done via a determination of the relative phase shift of the magnetic fluctuations collected by toroidally separated magnetic pick-up coils. In this paper, the method of determining the mode numbers on ASDEX Upgrade from magnetic pick-up coils is reviewed in section 2 and examples of mode determinations are presented to show the accuracy and the limits of the method. The significant improvement of the determination of mode numbers of high frequency fluctuations by considering intrinsic coil phases $[7\rfloor$ is demonstrated.

In section 3 a low ELM frequency discharge is used to identify toroidal mode numbers of different phases of ASDEX Upgrade inter-ELM oscillations. An estimation for the position of the inter-ELM oscillation from the rotation frequency is given and the usefulness of ELM synchronization in terms of characterization of inter-ELM oscillations is presented.

Section 4 includes a summary of the findings on inter-ELM modes together with a discussion on the localization and the mode numbers of the ELM associated phenomena ending with a short outlook.

\section{Mode number determination}

For the determination of toroidal mode numbers of coherent radial magnetic oscillations a toroidal ballooning pick-up coil array containing six coils with a sample rate of $2 \mathrm{MHz}$ is installed on ASDEX Upgrade, see Figure 1. The coils are placed inside the vessel along the outer midplane. The surface normal of each coil points to the geometric center of the torus and therefore they are measuring the temporal derivative of the radial magnetic field $\dot{\mathrm{B}}_{r}$. If the phases of a coherent magnetic oscillation with a frequency $\omega$ measured from each of the coils are known, the associated mode number can be calculated by a linear fit to the phases against the position $\phi$ of the coils. The phases $\varphi_{i}(\omega)$ of each coil $i$ can be determined from the Fourier transform $\hat{S}_{i}(\omega)$ of the coil signal $S_{i}(t)$ :

$$
\varphi=\arctan \left(\frac{\operatorname{Im}(\hat{S})}{\operatorname{Re}(\hat{S})}\right)
$$

To avoid edge effects in the Fourier analysis of very short time windows, which usually create noise close to $\omega=0$, it is reasonable to include proper windowing functions. In 

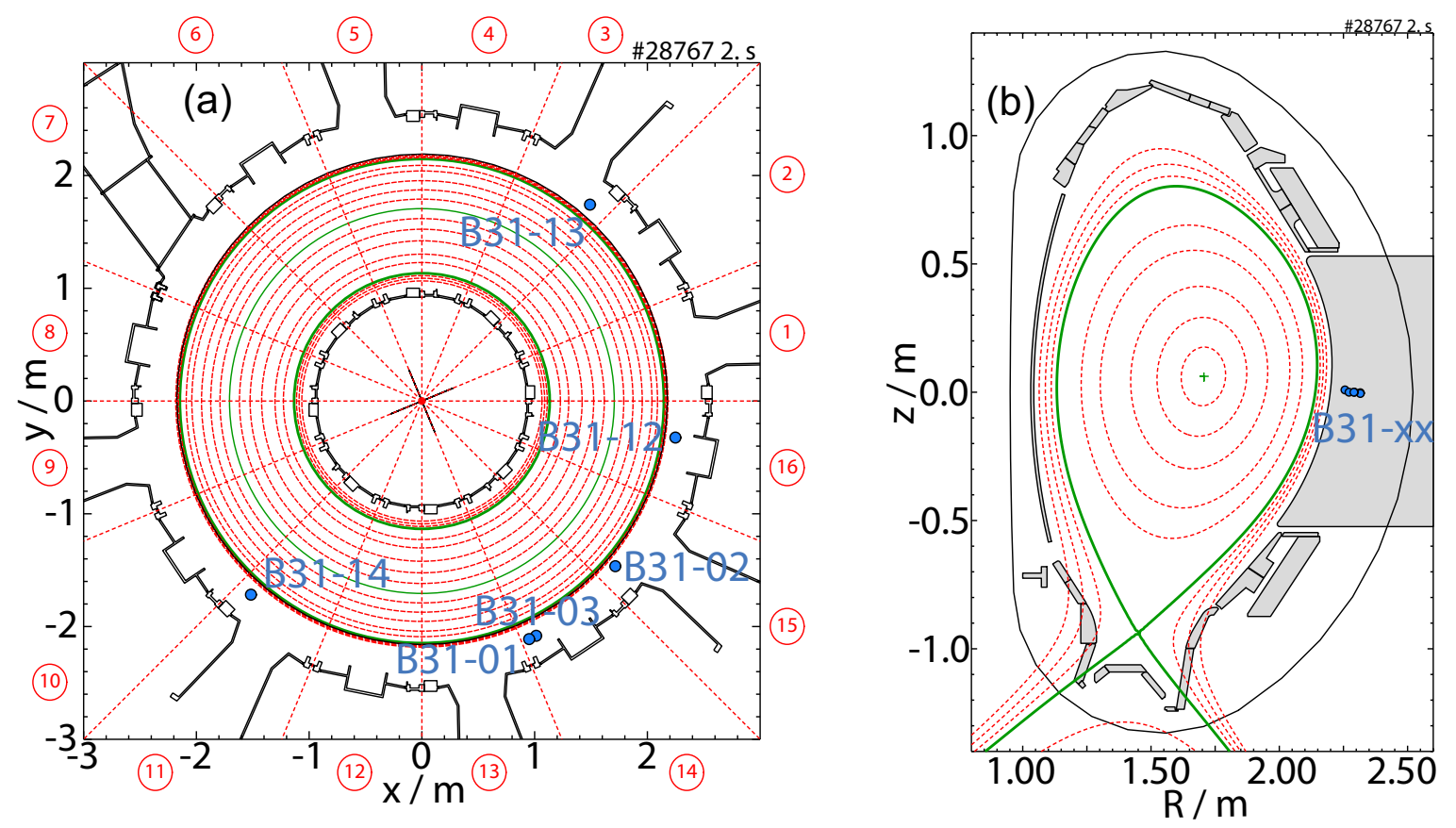

Figure 1. Position of the toroidally distributed ballooning pick-up coils at the outer midplane (a) in the toroidal and (b) mapped to a poloidal cross section of ASDEX Upgrade

this case a Gaussian windowing function $W(t)$ was used, which reduces the low frequency noise, without smearing out frequencies strongly:

$$
\hat{S}(\omega)=\sum_{j} S\left(t_{j}\right) W\left(t_{j}\right) \exp \left(i \omega t_{j}\right)
$$

Figure 2 shows a mode number determination fit. This fit was performed for a $10 \mathrm{~ms}$ time span where two $n=2$ and $n=3$ modes, rotating with the same velocity in the co-current direction, are present. The calculated phases are for both modes (with different $\omega$ ) plotted against the coil positions $\phi$ with blue circles. As the calculated phases have values in the range of $\varphi \in[-\pi, \pi)$ and the coils are covering a toroidal angle range of $\pi$, an $|\mathrm{n}| \geq 2$ mode will experience at least one phase jump of $2 \pi$ from one coil to the next. Therefore, it is necessary to allow shifts of the phases by $\Delta \varphi=k( \pm 2 \pi), k \in \mathbb{N}$, to calculate the absolute phase of each coil. The mode number is given by the slope of the best fitting line, calculated from a least square regression. In this context the sign of the slope determines the direction of the propagation in the laboratory frame. A positive (negative) slope is connected to a propagation in the ion(electron)-diamagnetic or co(counter)-current direction. In the presented case of two core modes the fit with a positive slope reproduces the data much better than with a negative slope.

Examples which show the precision of the determination of toroidal mode numbers are given in Figures 3 and 4 for two different discharges with different MHD phenomena present. The figures show first a time resolved frequency histogram (a). To create such a histogram a certain time interval which is given on the top of the plot is chosen and 


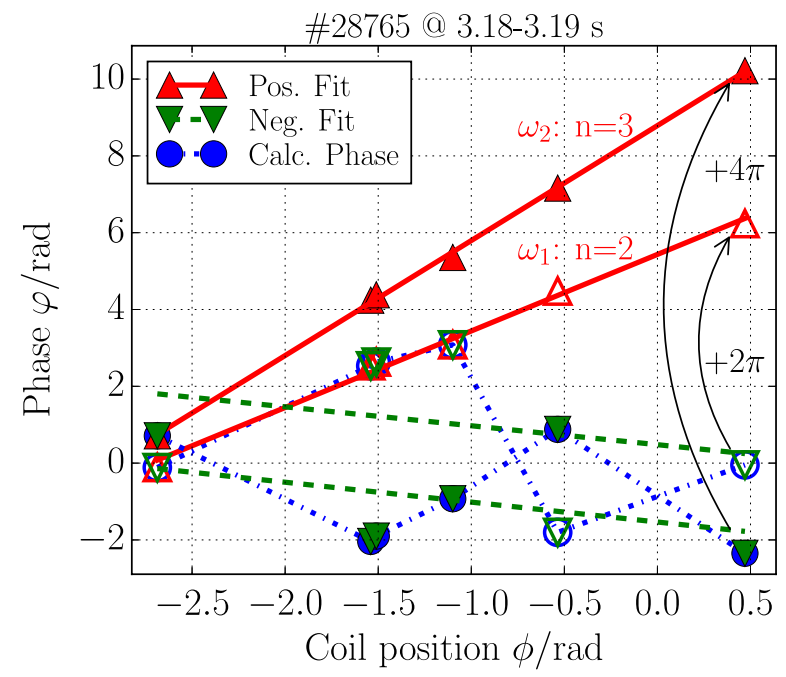

Figure 2. Linear fits to the calculated phases $\varphi(\omega)$ allowing shifts of $\Delta \varphi=k( \pm 2 \pi)$, $k \in \mathbb{N}$ for two $n=2\left(\omega_{1}\right.$, open symbols) and $n=3\left(\omega_{2}\right.$, closed symbols) modes measured by the six toroidally distributed pick-up coils at positions $\phi$

subdivided into sub-intervals to gain the temporal evolution. From all sub-intervals spectra for all six toroidal pick-up coils are calculated, binned together and normalized to the maximum intensity. This is the reason why it is worth calling it a frequency histogram and not only a spectrum. It shares both intensity scale and frequency axis with the second plot.

The second plots (b) are frequency resolved mode number histograms. To calculate these a time range with constant plasma conditions is selected, which is marked with white vertical lines in the frequency histogram (Fig. 3 and 4 (a)). This time window is then evaluated by the previously described linear fit method for each frequency, where the intensity is again the averaged intensity of all coils at the selected frequency. The histograms are normalized to the maximum intensity.

The third plots (c) show the raw signal of one of the magnetic pick-up coils which reflects the characteristic shape of the present mode.

The last plots (d) are frequency integrated mode number histograms. They are calculated from the frequency resolved mode number histograms (b) by integrating the intensities over all frequencies. Therefore it shares the mode number axis with the plot (b). The integration can also be restricted to a given frequency range to pronounce special aspects in the mode number histogram. If a certain range is chosen it is highlighted in the plot itself, otherwise integration is done in the whole frequency range of the frequency resolved mode number histogram.

In Figure 3 an $n=1$ core mode with higher harmonics of up to $n=9$ propagating with the core plasma rotation velocity is analyzed. The frequency histogram shows that the core mode rotation velocity increases during the shown time frame leading to a raise of the frequencies. For the frequency resolved mode number histogram, Figure 3 (b), a short time interval of $10 \mathrm{~ms}$ was chosen such that the frequency stays almost con- 

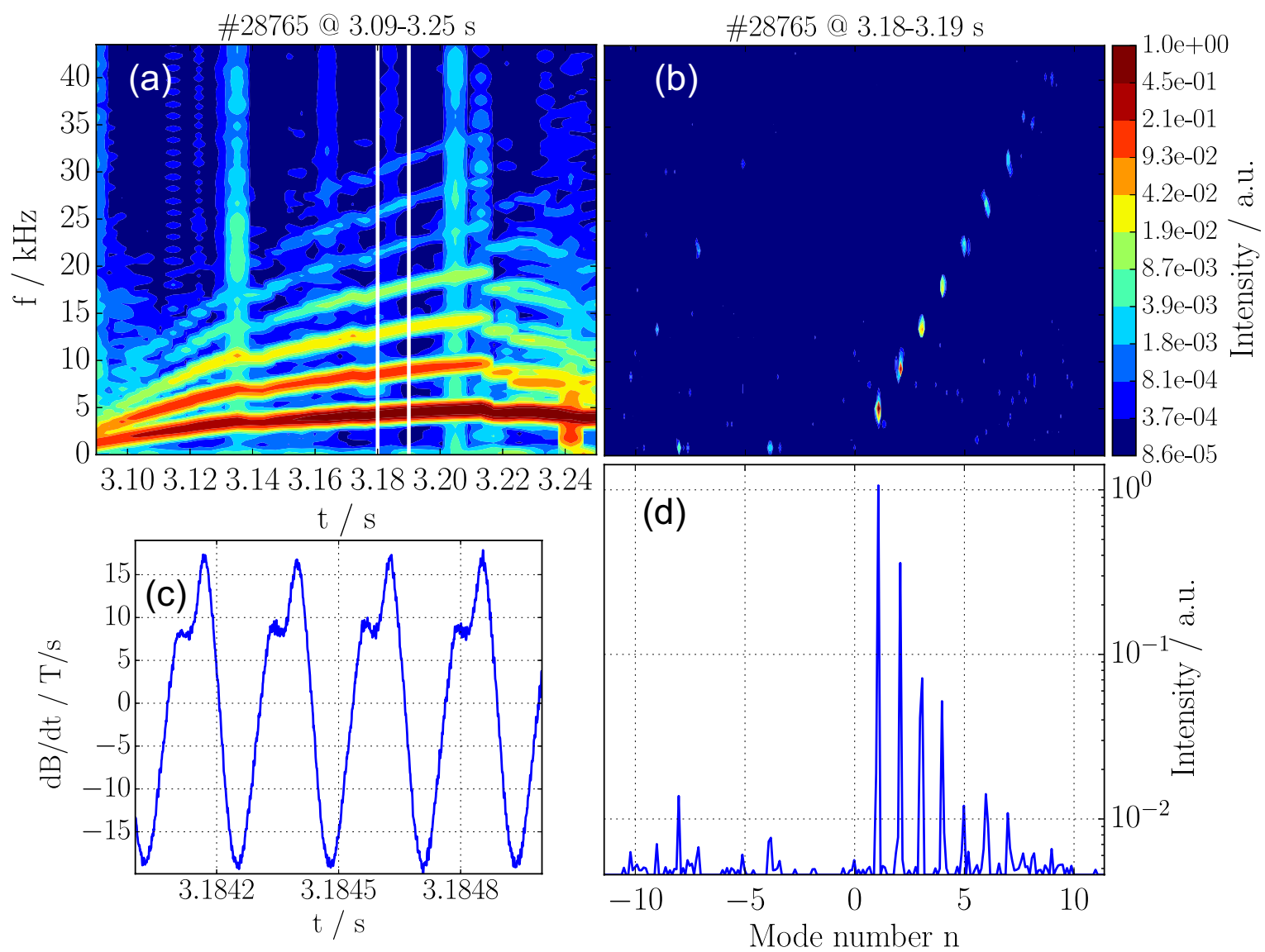

Figure 3. (a) Time resolved frequency histogram, (b) frequency resolved mode number histogram, (c) magnetic signal of the B31-14 coil and (d) a frequency integrated mode number histogram for an $n=1$ core mode propagating in the co-current direction and its higher harmonics

stant. The core mode with its higher harmonics form a straight line with its origin at $f=0 \mathrm{kHz}, n=0$ in this plot. The formation of such a straight line means that all of the structures $(n=1 \ldots 9)$ have the same $\mathrm{f} / \mathrm{n}$ value. They are most probably rotating with the same velocity and are therefore at the same radial position in the plasma. Such concise lines are called mode branches in the following. The integrated mode number histogram shows very precisely peaks at $n=1 \ldots 9$ but with an almost exponential decrease of intensity. The magnetic raw signal shows the characteristic structure of the $n=1$ oscillation together with some deformation caused by the much weaker $n=2$ component. The smaller structures are no longer visible in the magnetic signal because of their weak amplitude.

In Figure 4, an example of a so called edge snake [8] that has mode numbers $n=$ $-(1 \ldots 16)$ and rotates in electron-diamagnetic drift direction is evaluated. It appears on short time scales and is therefore only visible as a number of different frequencies in the frequency histogram between 2.995-3.025 s of this discharge. During the presence of the edge snake the magnetic raw signal shows a kind of bursty behavior which is a 

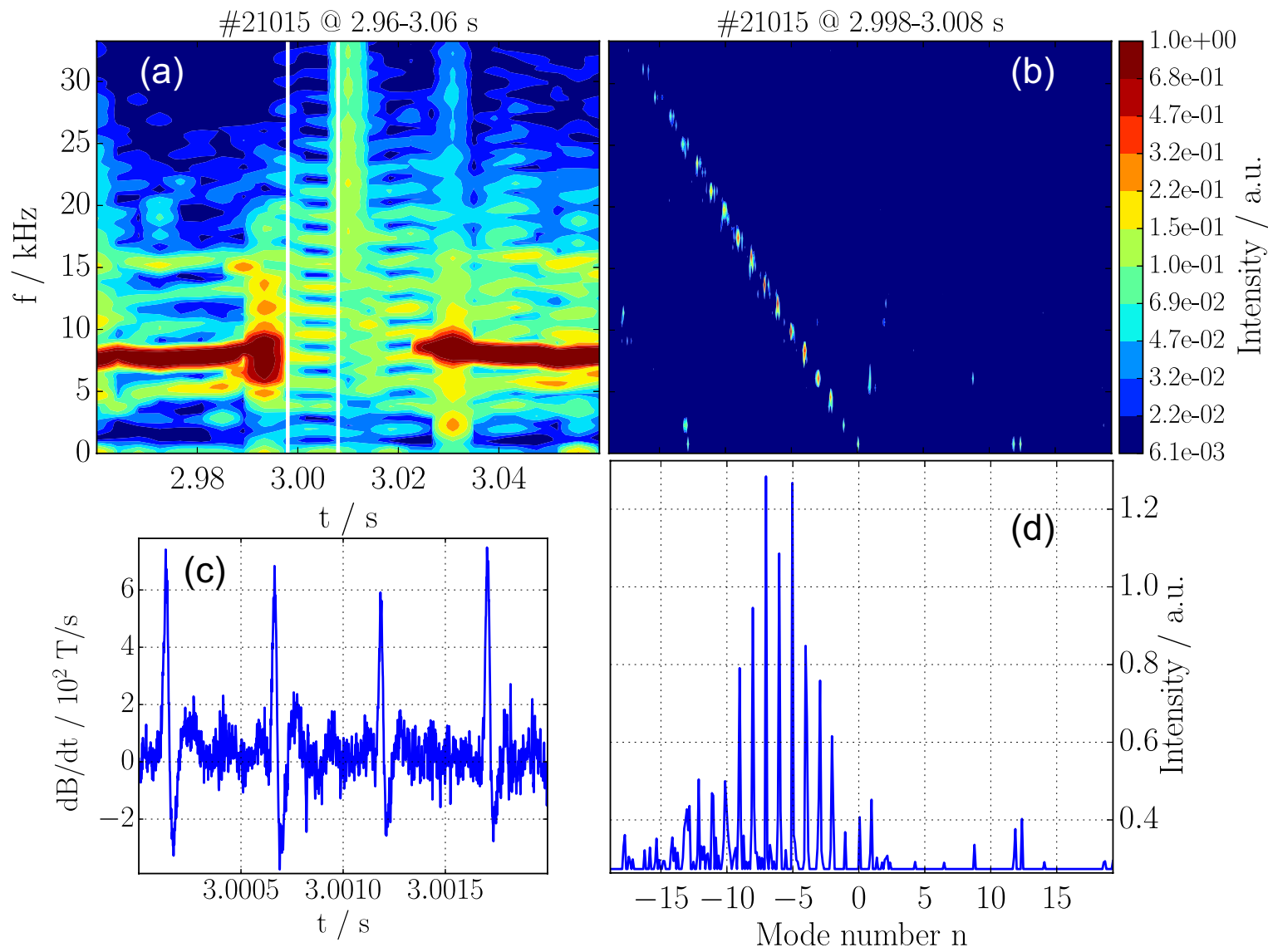

Figure 4. (a) Time resolved frequency histogram, (b) frequency resolved mode number histogram, (c) magnetic signal of the B31-14 coil and (d) a frequency integrated mode number histogram for an $n=-(1 \ldots 16)$ edge snake propagating in the electron diamagnetic direction.

result of superposed phase aligned oscillations with different frequencies with similar amplitudes. The mode number histogram shows again very clear peaks with the highest intensity at $n=-7$. In the frequency resolved mode number histogram again all substructures appear on a straight line through the origin, indicating that they build together one structure placed at one radial position.

As it can be seen for the two examples the method of determining toroidal mode numbers works with a high precision for mode numbers at least up to $n=16$. In principle the highest possible mode number that can be calculated with this method is given by the smallest distance between two coils. In the case of ASDEX Upgrade this is the distance $\Delta \phi_{3,1} \approx 0.05 \mathrm{rad}$ between the coils B31-03 and B31-01, which would be equivalent to a mode number in the range of $n= \pm \pi / 0.05 \approx \pm 62$. However, due to uncertainties in position and phase $n=62$ can not be detected. Nevertheless, it is possible to exclude the appearance of very high mode number fluctuations if the phase shift between these two closest coils is small.

As mentioned, one issue is the imprecise knowledge of the measured phase. It was 

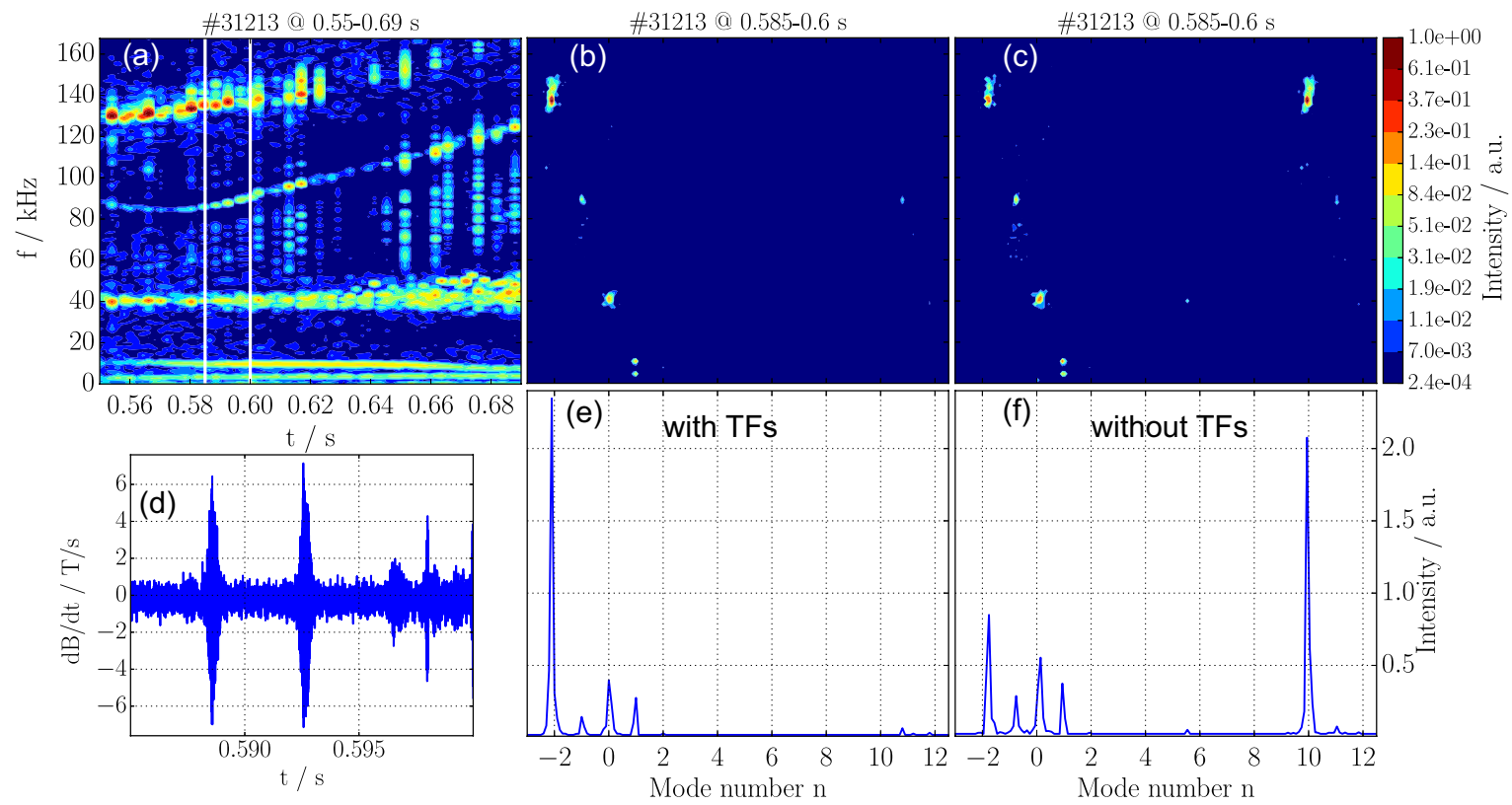

Figure 5. (a) Time resolved frequency histogram, (b) frequency resolved mode number histogram calculated with transfer functions (TFs) and (c) without, (d) magnetic signal of the B31-14 coil and (e) a frequency integrated mode number histogram calculated with TFs and ( $\mathrm{f}$ ) without for an $\mathrm{n}=1$ core mode with its harmonic, an $n=0$ GAM, and an $n=-1$ and $n=-2$ mode. Omission of TFs leads to significant misinterpretation of the $n=-2$ mode at $140 \mathrm{kHz}$

shown by Horvath et al. [7] that the installed coils experience intrinsic phase shifts due to induced eddy currents in the coil surrounding vessel structure or resonances in the probe or measuring circuit. The intrinsic phase shifts affect the mode number determination mostly for frequencies $f=50-250 \mathrm{kHz}$, where they reach values of up to 1 radian. Therefore, it was proposed to include frequency dependent phase transfer functions $\mathrm{TF}(\omega)$ for each coil to correct for this effect. This leads to a corrected phase $\varphi^{\prime}(\omega)=\varphi_{\text {meas }}(\omega)+T F(\omega)$.

Inclusion of the proposed transfer functions leads to a significant improvement of the determination of mode numbers especially in the mentioned frequency range. In Figure 5 a mode number determination of several mode structures was performed with and without transfer functions. Again a frequency histogram and mode number histograms are plotted together with the magnetic raw signal. The mode number histograms including the transfer functions are Figures $5(\mathrm{~b})$ and (e). The ones without transfer functions are Figures 5 (c) and (f). Clearly visible are an $n=1$ core mode at $f=5 \mathrm{kHz}$ and its harmonic at $f=10 \mathrm{kHz}$, an $n=0$ structure at around $f=40 \mathrm{kHz}$ which can be identified as an geodesic acoustic mode (GAM) $\mid 9\rfloor$ and two modes with $n=-1$ and $n=-2$ at $f=90 \mathrm{kHz}$ and $f=140 \mathrm{kHz}$ rotating in the electron diamagnetic direction. The magnetic raw signal for this time range shows the bursty behavior of the dominant high frequency $n=-2$ mode.

Results of the mode numbers of low frequency fluctuations like the core mode or the 
GAM are very reasonable also when the transfer functions and therefore the intrinsic coil phases are omitted. Mode numbers for high frequency fluctuations like the $n=-2$ mode at $f=140 \mathrm{kHz}$ can have big errors if the transfer functions are omitted, as in this case the $n=-2$ would be erroneously interpreted as an $n=+10$ mode. As ELM associated fluctuations have frequencies between $50-500 \mathrm{kHz}$ mode numbers of inter-ELM phenomena can only be reliably determined when transfer functions are taken into account.

Although a determination of mode numbers is very much improved by including transfer functions, phase fitting can give wrong results due to errors in the transfer functions or imprecise knowledge of the coil positions, especially for mode numbers that reflect the geometric distribution of the coils. One of these mode numbers is $n=-6$, which typically gives wrong fit results at $n=+6$ and vice versa which will be discussed later.

\section{Results for inter-ELM modes}

To resolve different phases during the ELM cycle an ASDEX Upgrade discharge (\#28767) with a stationary phase from 2.0 to 2.5 s containing 25 ELMs with low ELM frequency of about $f_{\mathrm{ELM}}=50 \mathrm{~Hz}$ was chosen. Other parameters are $I_{\mathrm{P}}=0.8 \mathrm{MA}$, $P_{\text {Heat }}=P_{\mathrm{NBI}}+P_{\mathrm{ECRH}}=2.5+1.5 \mathrm{MW},\left|B_{\mathrm{t}}\right|=2.5 \mathrm{~T}$ and line averaged core electron density $n_{\mathrm{e}}=6.0 \cdot 10^{19} \mathrm{~m}^{-2}$. Figure 6 shows time traces of the magnetic pick-up coil signal, the inner divertor shunt current, the ELM frequency, the plasma stored energy and the line averaged density at the core and the edge.

For a discussion of mode numbers of ELM associated phenomena appearing during the chosen discharge magnetic signals and kinetic profiles were ELM synchronized to get enough statistical information. ELM synchronization was previously done with respect to the $\mathrm{D}_{\alpha}$ light or the divertor shunt current signal $[10,11]$. For the determination of ELM synchronized magnetic coherent signals it was found to be more accurate to synchronize to the rising flank of one magnetic pick-up coil signal at the onset of the ELM.

Figure 7 presents ELM synchronized data points of the electron temperature $\mathrm{T}_{e}$ and density $\mathrm{n}_{e}$ at the pedestal top $(\rho=0.965$ and $\rho=0.98)$ evaluated with the Integrated Data Analysis tool (IDA) [12 and the inner divertor shunt current as a measure for the transport over the separatrix. The signals are synchronized to all 25 ELM crashes at $t=t_{\text {ELM }}$. Also plotted are smoothed signals of all quantities. From these signals one can identify several phases in the ELM cycles marked by the black vertical lines. These phases will later be related to the magnetic fluctuations. Further information on the connection between inter-ELM modes with pedestal profiles are given in $[6,13,14,15]$. Here only a short description of the phases is presented.

The density recovery phase (I) is found right after the ELM crash. Although the recovery starts already a bit earlier and therefore overlaps with the here defined ELM phase, this is the phase during which the density almost recovers from the crash. To- 


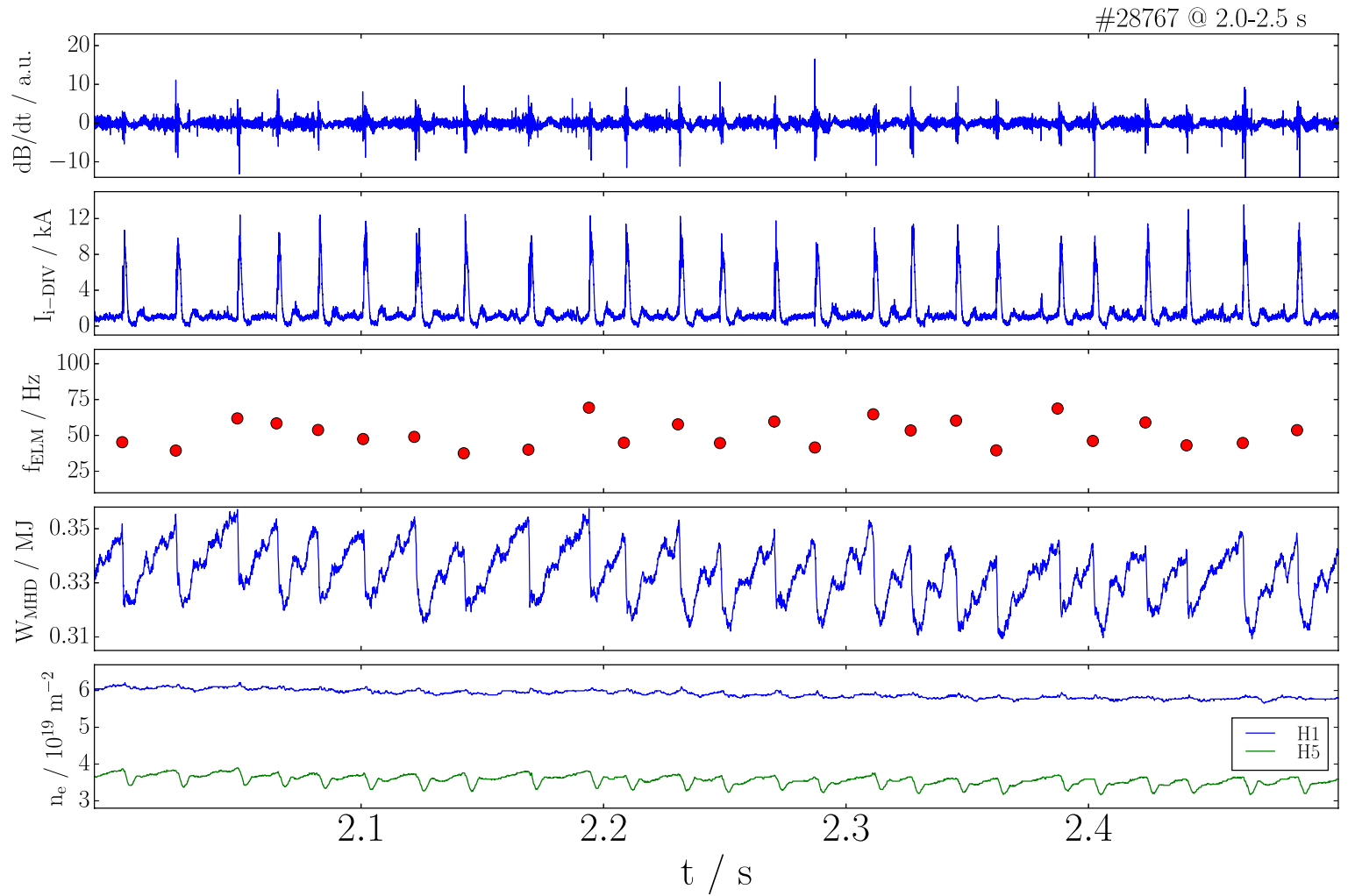

Figure 6. Time trace of the analyzed discharge \#28767. Shown are the signals of the magnetic pick-up coil B31-14, the inner divertor shunt current, the ELM frequency, the stored energy calculated from the equilibrium and the line averaged density measured from two interferometry channels through the core (H1) and the edge (H5)

gether with the density recovery the particle transport over the separatrix decreases. In this phase the electron temperature recovery is slow. As this phase has not yet reached strong gradients in the edge, the drive for modes in the edge is weak.

During the second phase after the ELM crash (II) the temperature recovery is increased and density reaches its maximum and starts to decrease again. Also the transport across the separatrix reaches its minimum first but starts to increase strongly at the end of this phase.

In the third phase after the ELM crash (III) the temperature recovery slows down again. At the beginning of this phase the pedestal density drops slightly which goes hand in hand with an increased transport visible as an increase of the divertor currents. This increased transport is slightly reduced towards Phase (IV) which also leads to a recovery of the pedestal density.

Phase (IV) after the ELM resets the temperature very slowly to its maximum value. The transport reaches an equilibrium value, which is much higher than the minimum reached in phase (II). The density at the pedestal top adjusts to similar values as in phase (III). It was shown by Laggner et al. [14] that the gradients of both quantities electron temperature and density are clamped during this phase. This phase mainly 


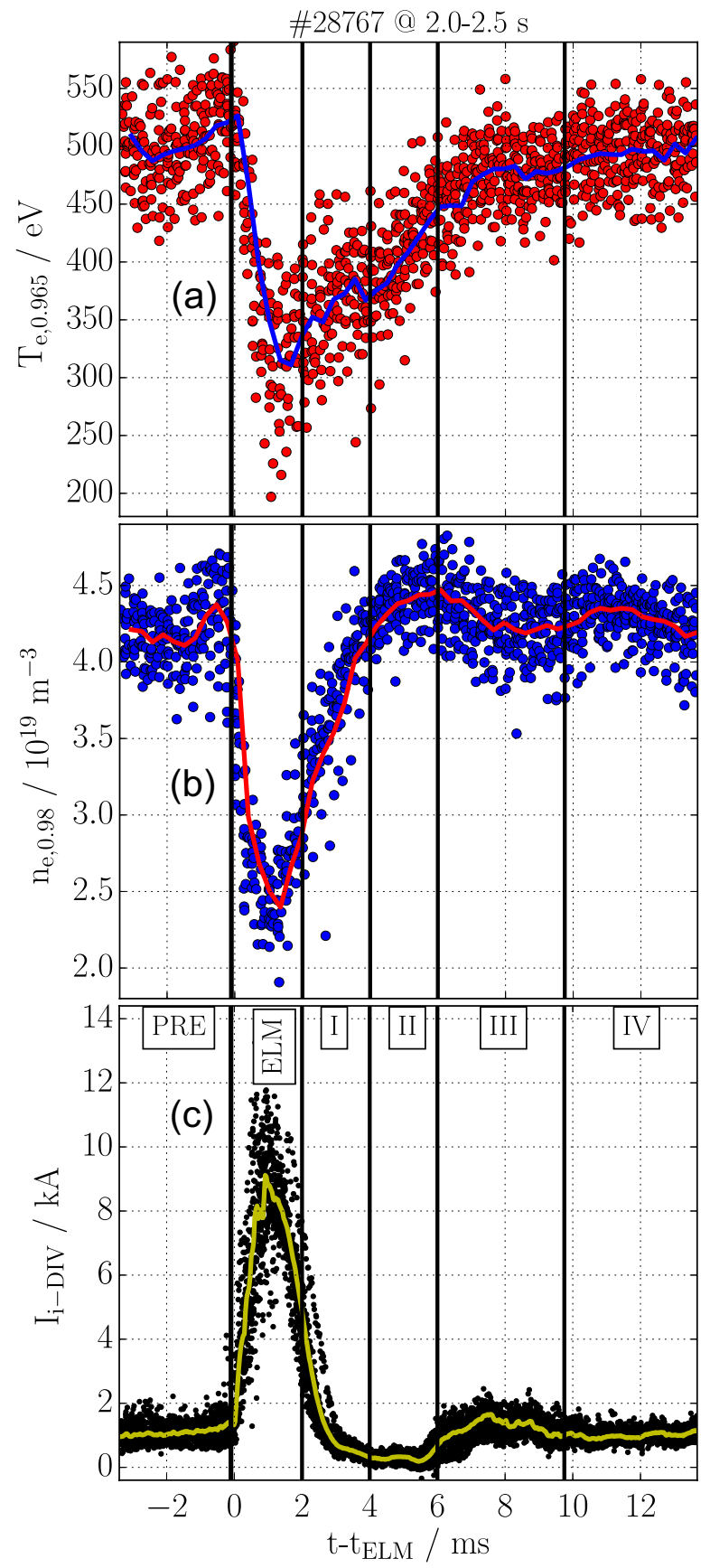

Figure 7. ELM-synchronized data points of (a) the electron temperature $\mathrm{T}_{e}$ and (b) density $\mathrm{n}_{e}$ at the pedestal top $(\rho=0.965$ and $\rho=0.98)$ evaluated with IDA and (c) the inner divertor shunt current as a measure for the transport over the separatrix together with the smoothed signal of all quantities. 
determines the length of the ELM cycle. It lasts until the phase before the ELM crash and is therefore identical with the beginning of pre-ELM phase.

This last phase before the ELM crash, or pre-ELM phase (PRE), includes a last slight increase of density and temperature and ends with the ELM crash.

The ELM crash phase (ELM) is defined by the strong losses of temperature and density which takes place on a millisecond time scale. It is important to mention that in the here defined phase already the density and temperature recovery starts. Nevertheless the transport of particles is also at the end of this phase very strong which is represented by the high divertor shunt current signal.

All these phases are slightly overlapping with each other and it is not easy to exactly determine an exact boundary. The phases defined here are a result of 25 averaged events which look different in detail. Nevertheless the profile recovery after each ELM is very similar up to $12 \mathrm{~ms}$ after the ELM onset and the starting point of each phase varies only about $0.5 \mathrm{~ms}$ for all 25 ELMs, which makes the ELM synchronization for the pedestal parameters a useful tool.

In the following the ELM synchronization of magnetic fluctuations are discussed. The synchronization of magnetic signals is done in the Fourier space to resolve participating frequencies. Figure 8 shows an ELM synchronized frequency histogram. It is similar to the previously explained time resolved frequency histograms with the difference that each time window with respect to one ELM crash is evaluated by a Fourier analysis for all six coils and the results are binned together in the time and frequency domain for all ELMs and normalized to the maximum intensity. The windows have a distance of $25 \mu \mathrm{s}$ and a length of $1 \mathrm{~ms}$ which creates an overlap of the time windows. The intensity of each participating frequency is again given by the Fourier coefficients of each time window. The resulting time resolved ELM synchronized frequency histogram is an indicator for the probability of different coherent signals with certain frequencies to appear during the whole time trace with respect to the ELMs. It is misleading to call it a time resolved ELM averaged spectrum, because it does not show an averaged ELM. More appropriate is the expression ELM synchronized intensity weighted frequency histogram.

In the ELM synchronized frequency histogram of the here analyzed discharge, see Figure 8 , one can differentiate phases by the appearance of different characteristic frequency bands which indicate the existence of modes. These phases are again the pre-ELM phase with two distinct frequency regions of increased mode activity at $0-100 \mathrm{kHz}$ and $200-$ $250 \mathrm{kHz}$, the ELM crash phase with strong broadly spread magnetic activity and the time after the ELM. One can identify the same four sub-phases after the ELM crash as in Figure 7, which are now characterized by their different magnetic activity. Phase (I) has only core MHD activity at $0-18 \mathrm{kHz}$. Phase (II) has magnetic activity in the frequency range up to $50 \mathrm{kHz}$ and weaker activity at higher frequencies. Phase (III) has dominant magnetic activity in the frequency range up to $150 \mathrm{kHz}$ and phase (IV) includes dominant modes in the frequency range of $200-250 \mathrm{kHz}$.

The ELM synchronized frequency histogram can not say something about the mode structure that appears before or after one single ELM. This is shown in the frequency 


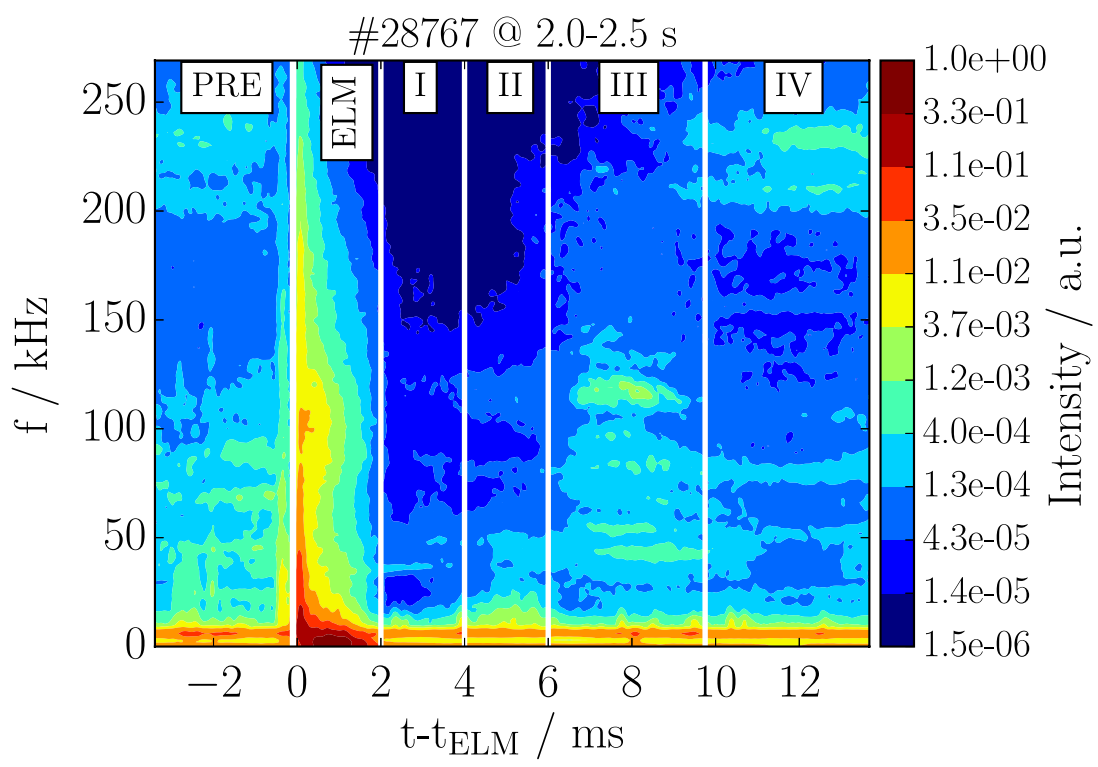

Figure 8. ELM synchronized frequency histogram with six distinct phases during the ELM cycle.

histogram of three single ELM events, Figure 9. Similar frequency bands as in the ELM synchronized histogram appear, but not all of them appear together like the ELM synchronized histogram could suggest. The first ELM has much more pre-ELM activity below $100 \mathrm{kHz}$ (red arrow). The second and third ELM have slightly different high frequency pre-ELM fluctuations at 240 and $210 \mathrm{kHz}$ respectively (white arrows). Therefore the ELM synchronized histogram can only be used to detect prevalent features but for conclusions on the connection of modes to ELMs a single ELM analysis is always needed. This will also be discussed in detail for the most critical pre-ELM phase.

The onset of different phases correlates with changes in the previously discussed kinetic profiles, see Figure 7. A toroidal mode number determination of phenomena during the ELM cycle was performed for all the phases separately. Therefore time windows with the length of $2.0 \mathrm{~ms}$ were chosen and analyzed in the following.

The most intense coherent magnetic signal throughout the ELM cycle in this discharge is produced by an $n=+1$ core mode at frequency $f_{\text {core } 1}=6.5 \pm 2 \mathrm{kHz}$ and its $n=+2$ harmonic at $f_{\text {core } 2}=13 \pm 4 \mathrm{kHz}$ rotating in current direction. In the following discussion this core mode will be omitted if not explicitly mentioned. All other coherent magnetic features are propagating in the electron diamagnetic direction, evolve during the ELM cycle and cause the frequency bands that are visible in Figure 8. These inter-ELM oscillations are the topic of the next paragraphs. 


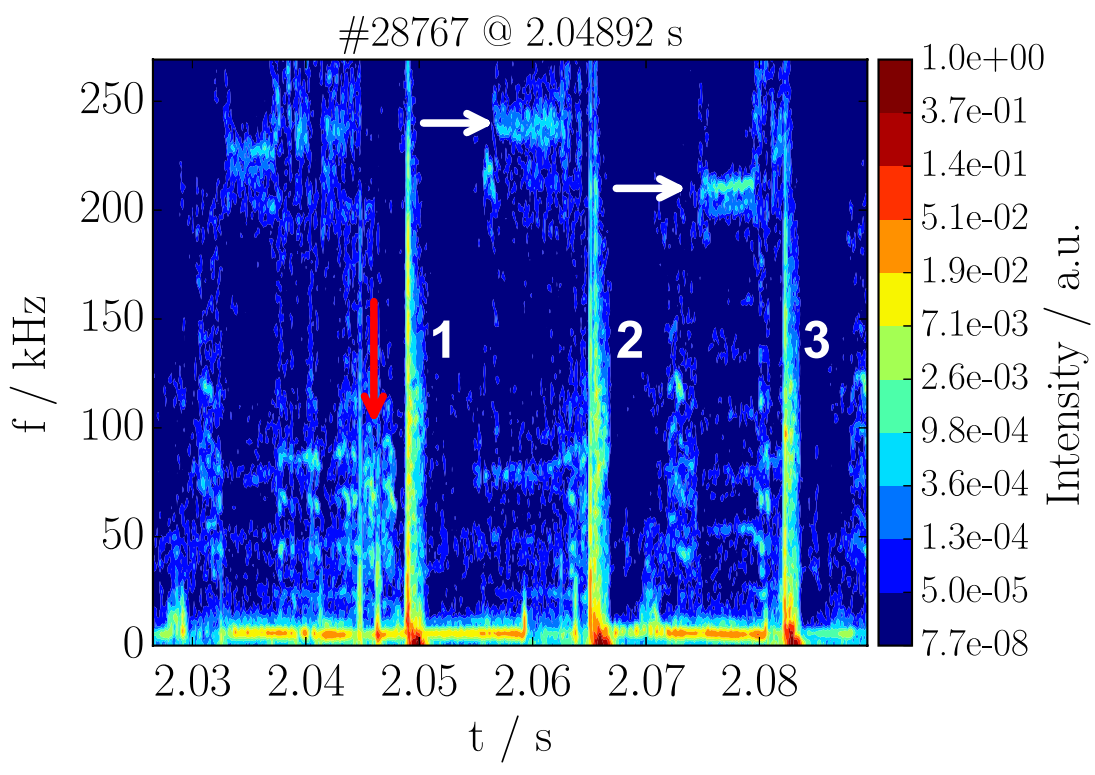

Figure 9. Time resolved frequency histogram of three single ELM events. The red arrow marks magnetic activity before the first ELM. White arrows mark different high frequency pre-ELM fluctuations at $240 \mathrm{kHz}$ and $210 \mathrm{kHz}$ before second and third ELM respectively.

\subsection{Phase I}

The contour plot in Figure 10 (a) shows an ELM synchronized frequency resolved mode number histogram. It is similar to the previously mentioned frequency resolved mode number histograms. The only difference is that here the not normalized frequency resolved mode number histograms of all $252 \mathrm{~ms}$ time windows around $3.0 \mathrm{~ms}$ after an ELM crash in the time between 2.0 and 2.5 seconds of the discharge are binned together and normalized to the maximum binned intensity. Also plotted are the mode number histograms integrated over all frequencies (b) and integrated over all frequencies higher than $18 \mathrm{kHz}$ (c). By integrating over frequencies higher than $18 \mathrm{kHz}$ the contribution to the histogram of the core mode is suppressed, which will be done in some of the following phases.

Phase I in the post-ELM phase exhibits almost no magnetic activity besides the core mode at $\mathrm{n}=1$ and $\mathrm{f}_{\text {core } 1}=6.5 \mathrm{kHz}$ in Figure 10 (a) and (b). This is the phase where the edge temperature and density gradients are crashed so that there is no drive for strong mode activity in the edge, see Figure 7. The only other magnetic activity is a weak coherent $\mathrm{n}=0$ structure with frequencies $\mathrm{f}=100-160 \mathrm{kHz}$ together with some noise, see Figure 10 (c). As this $n=0$ structure also appears in phase II and much more intense in the ELM-phase itself it will be discussed later. 


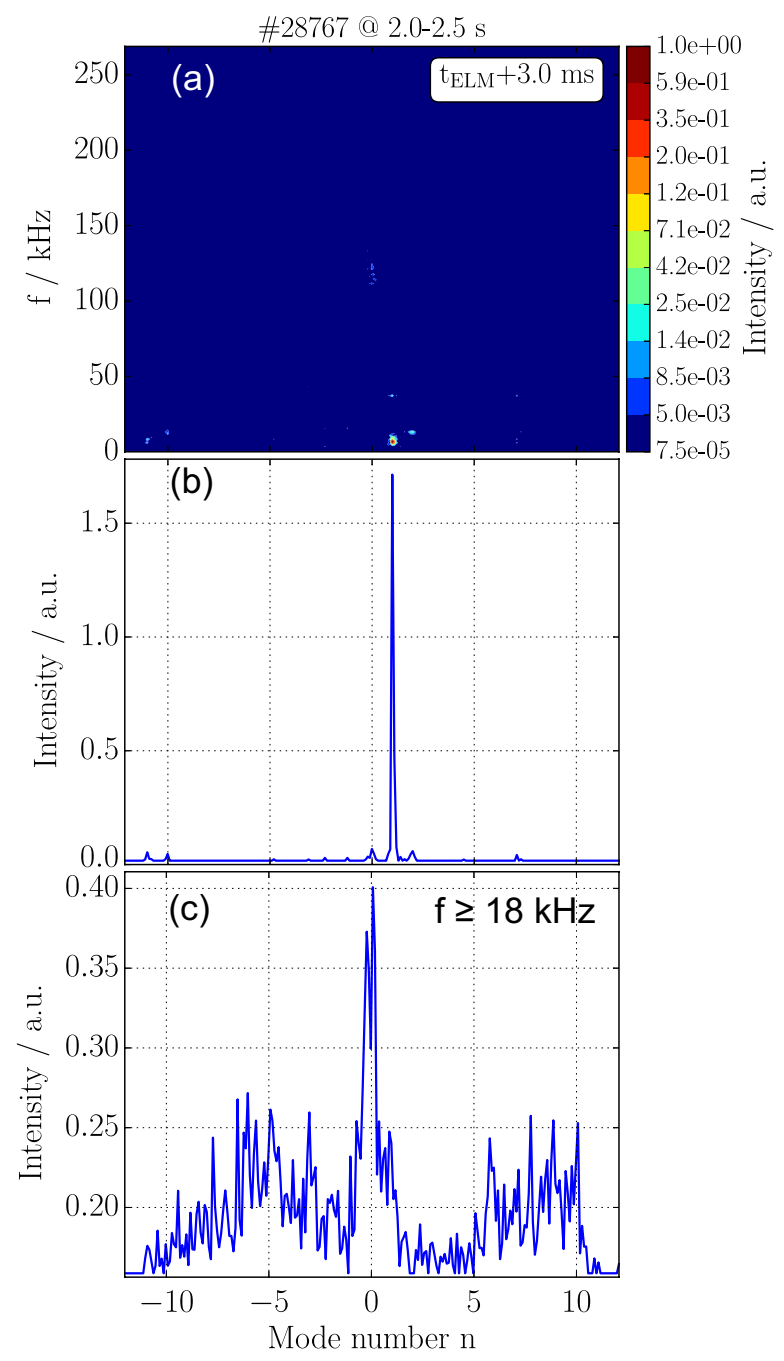

Figure 10. Phase I: (a) ELM synchronized frequency resolved mode number histogram and mode number histograms integrated (b) over all frequencies and (c) over frequencies above $18 \mathrm{kHz}$ of the 25 time windows around $3.0 \mathrm{~ms}$ after the ELMs.

\subsection{Phase II}

Figure 11 shows ELM-synchronized frequency resolved and frequency integrated mode number histograms for two time windows during phase (II). All of them are chosen such that they only include the first five ELMs in the given time trace because synchronization over more ELMs leads to a strong smear of mode numbers and frequencies. During this phase low frequency magnetic activity with not clearly defined negative mode numbers, Figure 11 (a) and (c), evolves into distinct modes in the range of $n=-(1 \ldots 8)$, Figure 11 (b) and (d). The transition time varies about $0.5 \mathrm{~ms}$ from ELM to ELM. This leads to a blur of both structures if evaluated for all 25 ELMs.

Together with the low frequency fluctuation again an $n=0$ structure is visible in both time frames. Also a weak branch of mode numbers at high frequencies with $n=-7,-8$ $(f \approx 180,210 \mathrm{kHz})$ begins to form at the end of this phase and will evolve towards 

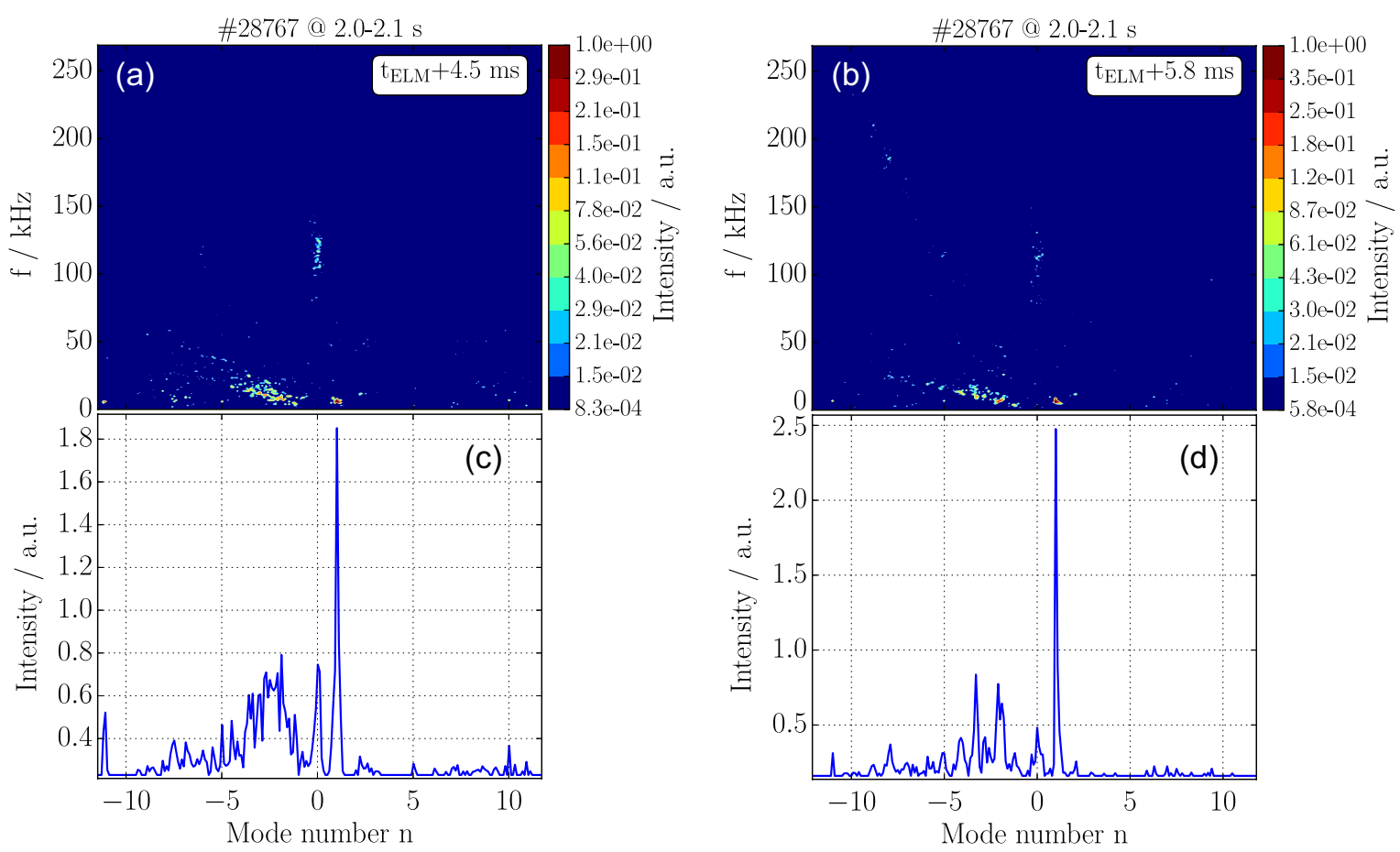

Figure 11. Phase II: (a),(b) ELM synchronized frequency resolved mode number histograms and (c),(d) mode number histograms integrated over all frequencies of the five time windows around $4.5 \mathrm{~ms}$ and $5.8 \mathrm{~ms}$ respectively after the five ELMs between $2.0-2.1 \mathrm{~s}$.

$n=-5$ and become dominant in phase (III). Even in a single ELM analysis both the low and the high frequency branch coexist at the end of this phase on the millisecond time scale given by the evaluated window length.

At the end of this phase the temperature at the pedestal top is still low whereas the pedestal top density reaches its maximum and starts dropping from there. This is the time point of the formation of the discrete high and low frequency structure which goes hand in hand with the transport increase in Figure 7.

\subsection{Phase III}

In phase III around $7.5 \mathrm{~ms}$ after the ELM crash three clear branches of modes are present. The onset of these modes coincides with an increased transport and a small drop in the pedestal density, see Figure 7. The branches form three different straight lines in the frequency resolved mode number histogram, Figure 12 (a), and have very sharply defined mode numbers in the frequency integrated mode number histogram, Figure 12 (b). All branches coexist on the $2 \mathrm{~ms}$ time scale and are not a result of the ELM synchronization which can be validated by single ELM analysis. The different slopes of the mode branches indicate again their different rotation velocities. Therefore the first branch (white dashed line) with mode numbers $n=-(1 \ldots 7)$ with frequencies $f=15-80 \mathrm{kHz}$ is rotating with a comparatively low velocity, whereas the second (green 


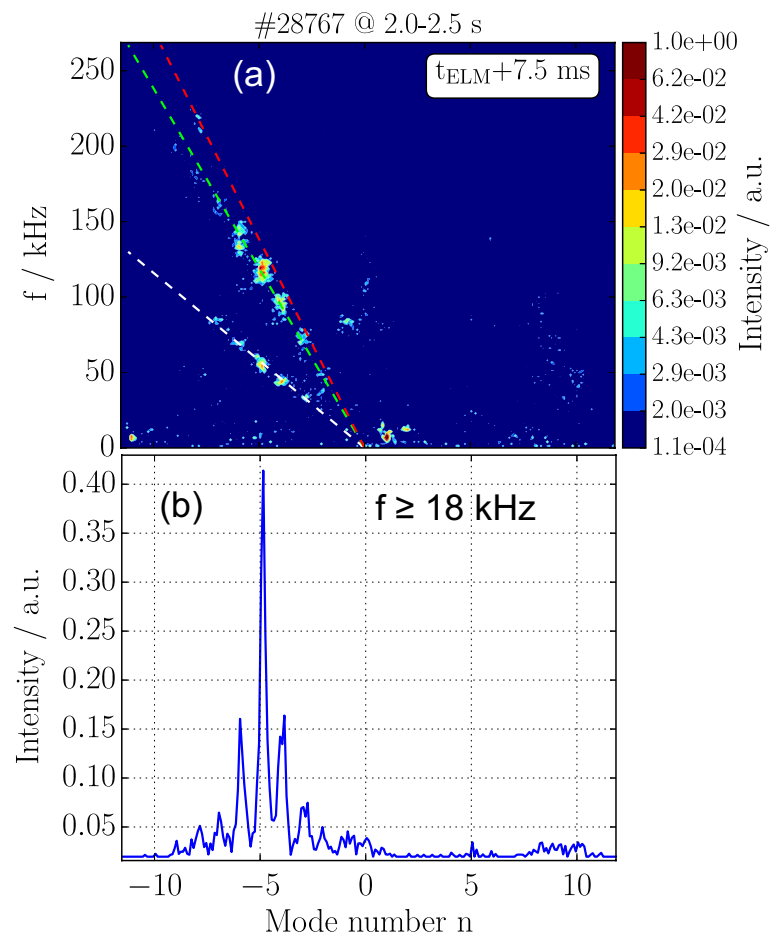

Figure 12. Phase III: (a) ELM synchronized frequency resolved mode number histogram and (b) mode number histogram integrated over frequencies above $18 \mathrm{kHz}$ of the 25 time windows around $7.5 \mathrm{~ms}$ after the ELMs. Red, green and white dashed lines indicate mode branches with different $\mathrm{f} / \mathrm{n}$ values.

dashed line) and most dominant branch $(n=-(1 \ldots 9), f=15-200 \mathrm{kHz})$ and the third (red dashed line) branch $(n=-8, f=220 \mathrm{kHz})$ have higher ones. In the following this velocity is used for a rough estimation of the localization of the modes. As already mentioned the second branch starts with $n=-7,-8$ already in phase II whereas the other branches show up in this phase first.

As it was shown by Laggner et al. [14」 the here discussed inter-ELM modes are placed in the strong pressure gradient region close to the plasma boundary. Some of the following assumptions used for the localization are only valid in this edge region. The mode velocity in the laboratory frame is given by the sum of toroidal and poloidal plasma rotation and its intrinsic toroidal and poloidal phase velocity:

$$
\vec{v}_{\text {Mode }}=\vec{v}_{\text {pol }}+\vec{v}_{\text {tor }}+\vec{v}_{\text {pol,ph }}+\underbrace{\vec{v}_{t o r, p h}}_{\approx 0} .
$$

The phase velocity $v_{p h}$ of modes in the plasma frame is either zero or mainly in poloidal direction as it moves with the diamagnetic velocity [16]. Therefore its toroidal part can be neglected in this equation and the total phase velocity is comparable to its poloidal part $v_{p h} \approx v_{p o l, p h}$. Therefore the poloidal projection $v_{\text {Mode,pol }}$ of the mode velocity is given by:

$$
v_{M o d e, p o l}=v_{p o l}+v_{p o l, p h} \approx v_{p, E \times B}+v_{p h},
$$




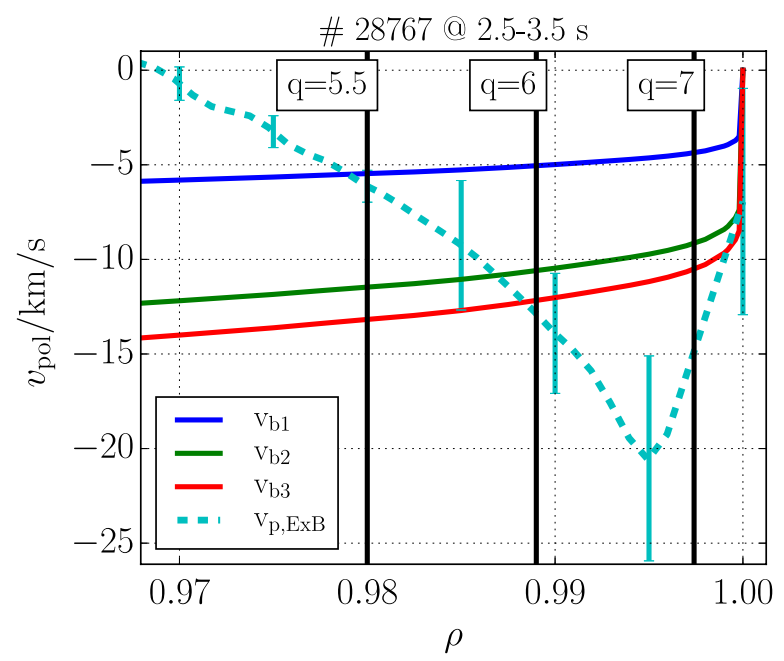

Figure 13. Comparison of the velocities of the three mode branches appearing around $7.5 \mathrm{~ms}$ after the ELM crash, Equation 5, and $v_{p, E \times B}$ measured by CXRS.

where $v_{p, E \times B}<0$ is the poloidal part of the $E \times B$ velocity, which is the main drive of the poloidal plasma rotation at the edge. The measured frequency of the mode is given by the sum of the toroidal and poloidal mode velocities divided by the toroidal and poloidal periodicities:

$$
f=\underbrace{\frac{n v_{\text {Mode,tor }}}{2 \pi(R+r)}}_{\approx 5 \mathrm{kHz}}+\frac{m v_{\text {Mode,pol }}}{2 \pi r}
$$

With $R=1.65 \mathrm{~m}$ being the major radius, $\mathrm{r}$ the radial position and $\mathrm{m}, \mathrm{n}$ the poloidal and toroidal mode numbers. For ASDEX Upgrade H-modes the toroidal plasma rotation close to the edge is about $v_{t o r}=5-20 \mathrm{~km} / \mathrm{s}[17,18]$. For the dominant $n=5$ the toroidal contribution to the frequency is therefore $2-8 \mathrm{kHz}$, with $r=0.5 \mathrm{~m}$. In this phase of the ELM cycle the $n=5$ is measured with frequencies of 50 and $120 \mathrm{kHz}$, see Figure 12 (a). Therefore the contribution of the toroidal mode velocity to the frequency can be neglected. The poloidal mode velocity can then be determined from the measured frequency via $v_{M o d e, p o l}=2 \pi r \frac{f}{q n}$, if the mode is close to the plasma edge and placed on a rational surface $q=m / n$.

The phase velocity can now be calculated from the mode velocity, given by the measured $\mathrm{f} / \mathrm{n}$ values and the poloidal part of the $E \times B$ velocity $v_{p, E \times B}(r)=\frac{\vec{E}(r) \times \vec{B}_{t}(r)}{B_{t}(r)^{2}}$ via Equation 4 , where the radial electric field $E$ is measured with the charge exchange recombination spectroscopy (CXRS) diagnostic $[18,19]$. Conversely, the mode can be localized with this equation if the phase velocity is known.

Figure 13 shows the comparison of both quantities, the poloidal mode velocity and $v_{p, E \times B}$, depending on $\rho_{p o l}$ together with the position of the rational surfaces $q=5.5,6,7$ for this discharge. For this comparison the three dominant branches with $f / n$ values of $f / n \approx-11.2,-23.5,-27 \mathrm{kHz}$ were chosen which are given by the slopes of the dashed 
lines in Figure 12. From this velocity comparison different radial positions of the modes are possible.

If the modes do not have any phase velocity (e.g. ideal peeling mode) they would be localized at the position where the mode velocity and $v_{p, E \times B}$ coincide. This happens either at $q=5.5-6$ or close to the separatrix $(\rho=1.0)$.

If the phase velocities of the modes would be the ion diamagnetic velocity, $v_{p h}>0$ (e.g. kinetic ballooning modes), the modes are localized where the mode velocity lies above $v_{p, E \times B}$ in Figure 13. As the absolute value of diamagnetic velocity and $E \times B$ velocity are almost the same near the $E \times B$ minimum [20], both quantities should even compensate and the mode velocity vanishes. This is at none of the shown positions the case, but because of the big uncertainties near the separatrix they can not be excluded from that region.

If the phase velocities of the modes would be the electron diamagnetic velocity, $v_{p h}<0$ (e.g. micro tearing modes), the modes are localized where the mode velocity lies below $v_{p, E \times B}$ in Figure 13. This is either the case at $q<6$ or very close to the separatrix, where uncertainties of $v_{p, E \times B}$ are so large that it is not possible to put a quantitative statement.

From this analysis it could be consolidated that the inter-ELM modes rotation velocity fits to the $E \times B$ velocity close to the maximum gradient but it is not possible to

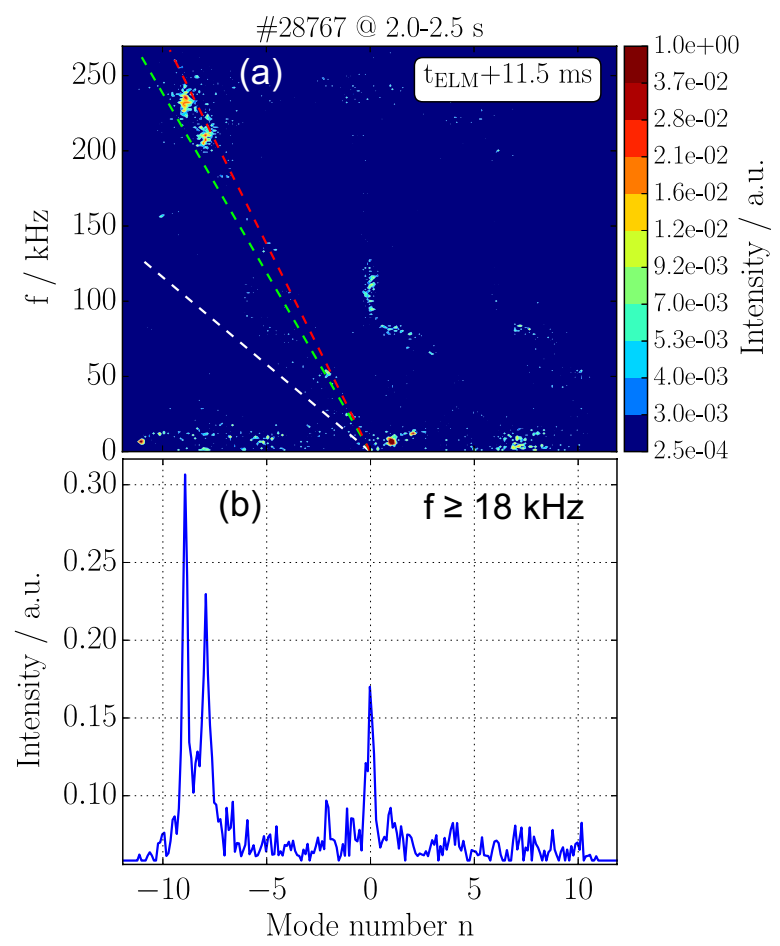

Figure 14. Phase IV: (a) ELM synchronized frequency resolved mode number histogram and (b) mode number histogram integrated over frequencies above $18 \mathrm{kHz}$ of the 25 time windows around $11.5 \mathrm{~ms}$ after the ELMs. Red, green and white dashed lines have the same slopes as in Figure 12 (a). 
determine an exact position or the exact phase velocity due to the uncertainties in the measurement of the radial electric field. Therefore an analysis of the poloidal mode number $m$ is necessary to determine the exact $q=m / n$ position.

\subsection{Phase IV}

In phase IV around $11.5 \mathrm{~ms}$ after the ELM crash the high frequency branch $(f=$ $200-250 \mathrm{kHz}$ ) of modes grows. There are still some medium frequency fluctuations present but it is difficult to say whether they belong to the medium or the high velocity branch, see Figure 8.

In the frequency resolved mode number histogram of this phase, Figure 14 (a), again the dashed lines with the same slope as in phase III are included to show that it is not the rotation velocity that is changing very much but the mode numbers and therefore the frequency given by Equation 5. The most dominant mode numbers are $\mathrm{n}=-8,-9$ during this phase, see Figure 14 (b). The onset of these modes coincides with the saturation of the electron temperature, a slight drop down of the pedestal density and the establishment of a transport equilibrium, see Figure 7. As this phase has a stronger pressure gradient and the mode numbers are higher than in phase III it might be that these modes are more pressure driven than the dominant ones in phase III.

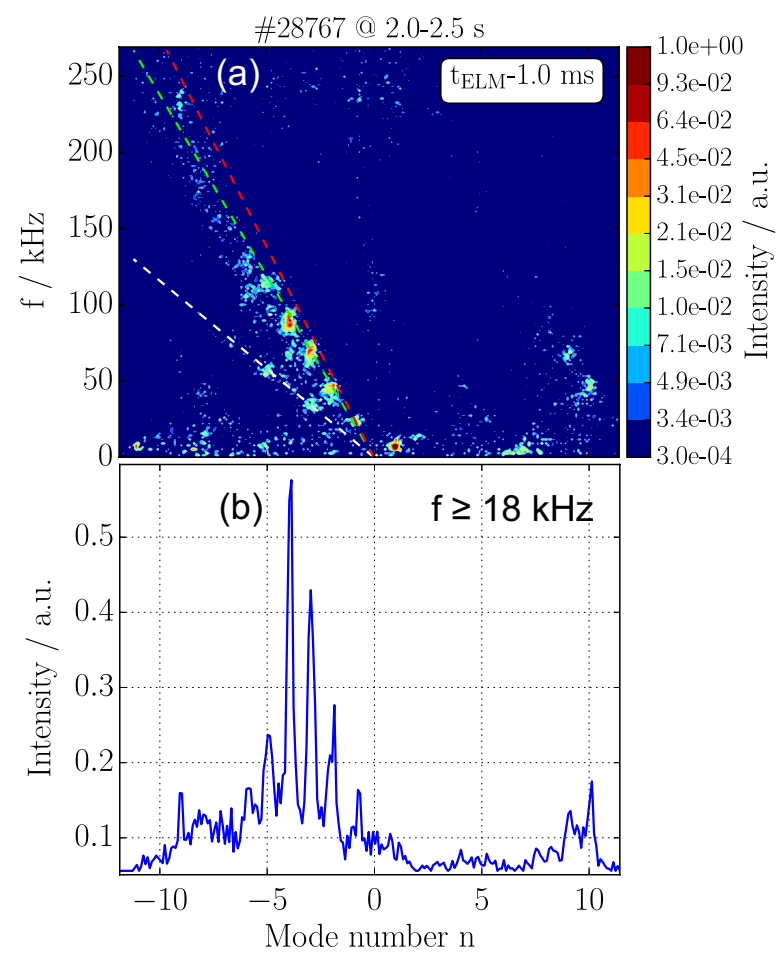

Figure 15. Pre-ELM phase: (a) ELM synchronized frequency resolved mode number histogram and (b) mode number histogram integrated over frequencies above $18 \mathrm{kHz}$ of the 25 time windows around $1.0 \mathrm{~ms}$ before the ELMs. Red, green and white dashed lines have the same slopes as in Figure 12 (a). 

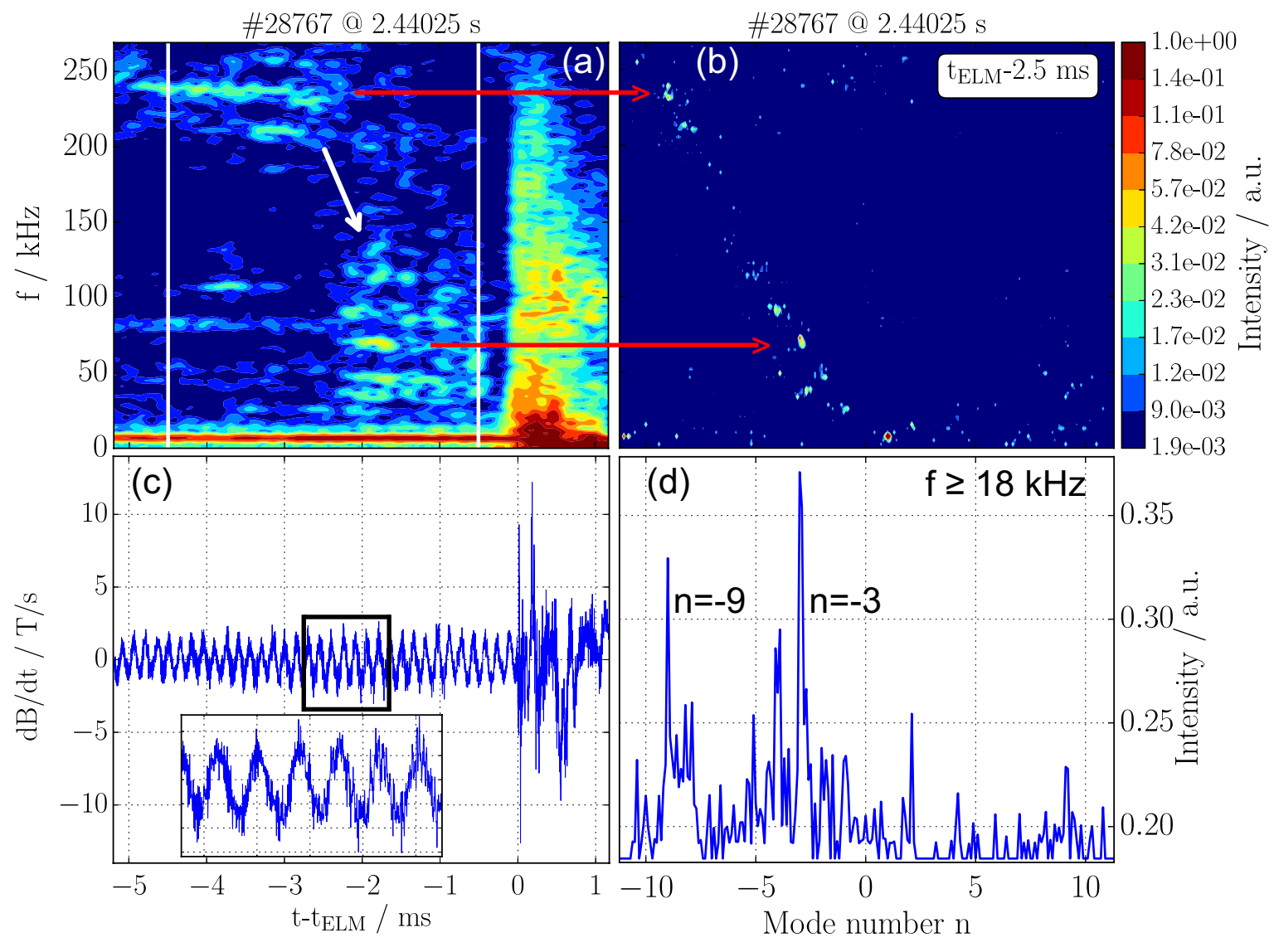

Figure 16. (a) Frequency histogram synchronized to one single ELM at $2.44 \mathrm{~s}$. The jump in the evaluated frame (marked with white stripes) from high frequencies to low frequencies is illustrated by the white arrow. (b) Frequency resolved mode number histogram and (d) mode number histogram integrated over frequencies above $18 \mathrm{kHz}$ of a $4 \mathrm{~ms}$ time window around $2.5 \mathrm{~ms}$ before one characteristic ELM onset. (c) The magnetic raw signal of the analyzed time frame together with a zoom around $2.2 \mathrm{~ms}$ before the ELM.

\subsection{Pre-ELM phase}

In the Pre-ELM phase around $1.0 \mathrm{~ms}$ before the ELM crash the same modes as for the phases III and IV are present with similar rotation velocities, but most dominant mode numbers decrease to $\mathrm{n}=-2,-3,-4$, which is visible in the ELM synchronized mode number histogram in Figure 15. Such a jump of mode numbers and therefore frequencies takes place just before the ELM crash. To see this mechanism more clearly one characteristic ELM was chosen from the time trace and analyzed. A frequency histogram, and the frequency resolved and frequency integrated mode number histograms of this single ELM are shown in Figure 16 together with the ELM synchronized magnetic raw signal. From the frequency histogram it is visible that the transition from higher to lower frequency takes place roughly $2.2 \mathrm{~ms}$ before the ELM and that the transition is such that the high 
frequencies stop abruptly and the $30-120 \mathrm{kHz}$ fluctuations appear again. This is also visible in the zoomed part of the magnetic raw signal, where high frequency oscillations disappear around $-2.2 \mathrm{~ms}$. This effect of the jump of mode numbers before the ELM onset is for almost all ELMs in the whole time trace very pronounced. For a few cases mode numbers even jump back and forth ending with lower frequencies before the ELM. Only broadband activities (like before the first ELM in Figure 9) can blur this effect. The mode number histograms are calculated for a $4.0 \mathrm{~ms}$ time window around $2.5 \mathrm{~ms}$ before the ELM crash to calculate mode numbers for both the high frequency and the low frequency domain. From the mode number histogram it can be seen that the lower frequencies just before the ELM onset are not caused by a slowing down and therefore a decrease of the velocity but a jump in the mode numbers. In this case the dominant mode numbers go from $n=-9$ to $n=-3$.

These results of a fast transition from high mode numbers to low ones in the pre-ELM phase fit qualitatively good to previous results from the non-linear MHD code JOREK $[21,22]$.

\subsection{ELM phase}

The ELM phase is defined here as the $2 \mathrm{~ms}$ long phase starting at the ELM crash. The mode number histogram in Figure 17 shows that the most dominant structure during this phase is a continuous negative $\mathrm{n}$ structure with frequencies less than $25 \mathrm{kHz}$ which peaks around $n=-3$, similar to the structure in phase II after the crash. Although the structure does not occur with single isolated peaks in the mode number histogram, it is clear that it is still a coherent structure. The exact structure is hard to determine either from the ELM synchronized or from a single ELM mode number determination because of the very short time intervals and the high noise. It is possible that the ELM consists out of a real $n=2,3,4$ structure rotating slowly in the electron diamagnetic direction but smeared out due to the very short time scales and therefore bad resolution in the Fourier analysis. Another possibility to interpret the continuity in the mode number histogram is that a evolution to a more Gaussian like toroidal shape has taken place so that the pure sinusoidal structure is lost. Nevertheless it must be pointed out that this structure looks very much like the structure that appears just before the ELM crash in the Pre-ELM phase with dominant structure at $n=-2,-3,-4,-5$ but here with strongly decreased velocity. As the radial electric field also collapses during ELMs [18], the reduced $E \times B$ velocity is mirrored in the lower frequencies of the mode.

In addition to the continuous negative $\mathrm{n}$ structure a structure around $80-130 \mathrm{kHz}$ is visible which still exists in the phases I and II after the ELM. In the mode number histogram including only frequencies higher than $50 \mathrm{kHz}$, see Figure 17 (c), it is visible that mode numbers of this structure are $n=0$ and $n= \pm 6$. It is not completely understood how to interpret these results. In order explain this phenomenon, modes with $n=-6$ and broadly spread frequency were simulated and evaluated with the same linear fitting algorithm considering the given geometry of the coils. These simulated 

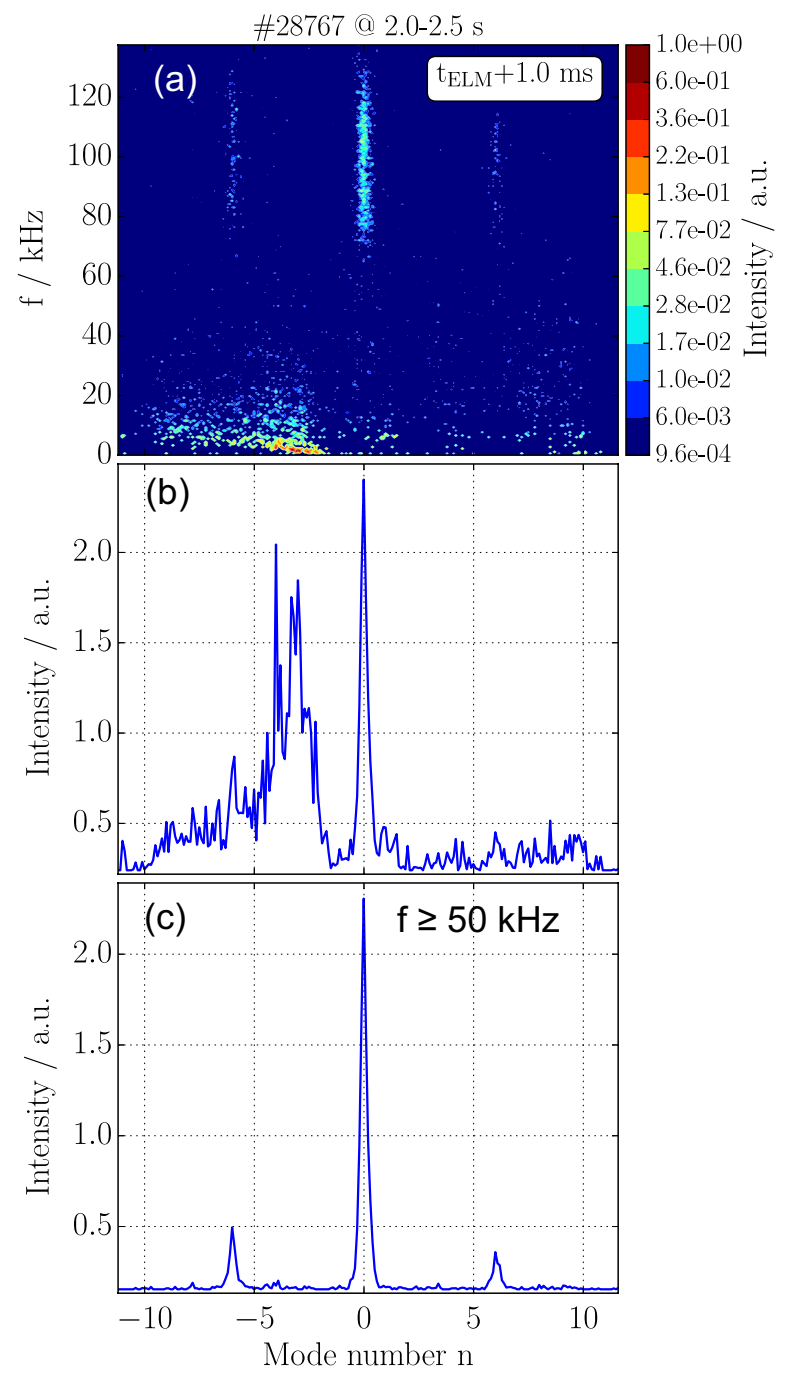

Figure 17. ELM phase: (a) ELM synchronized frequency resolved mode number histogram and mode number histograms (b) integrated over all frequencies and (c) over frequencies above $50 \mathrm{kHz}$ of the 25 time windows around $1.0 \mathrm{~ms}$ after the ELMs.

$n=-6$ modes showed always weaker artifacts at $n=+6$ and $n=0$. On the other hand a pure $n=0$ mode structure was simulated and it did not show any other structure beside the simulated $n=0$ also when adding a high noise level.

In addition to the simulation of modes a second evaluation of this phase with dropping the B31-14 coil and therefore changing the geometric arrangement was done. This evaluation still resulted in an $n=0$ together with $n=-6$ but it changed the dominant positive mode numbers, which suggests that the positive value is an artifact. Therefore the interpretation points into the direction that there exists some structure with a mode number $n=-6$ and the dominant $n=0$ structure but no structure rotating in the ion diamagnetic direction.

The broad spread in frequency is thought to be caused by a movement of the structure perpendicular to the field lines towards the $\mathrm{B}_{r}$-coils, first of all by the movement itself 
and secondly because the structure crosses regions with different plasma rotation velocities yielding also different frequencies.

The origin of the $n=0,-6$ stays unexplained. It might be that the strong non-linear interaction triggers a mode, e.g. a GAM with $n=0$, what would mean that the $n=-6$ is artificial. Another explanation would be that the strong exhaust during the crash causes a shaking of the plasma, which could also appear as an $n=0$ fluctuation. Another possible explanation would be the often described filaments that appear at the end of ELMs. Usually they are assumed as current carrying [23」 field aligned blob like structures which propagate in the scrape-off layer towards the $\mathrm{B}_{r}$ coils $[24,25]$. This fits to the here detected broad spread in frequencies and to the additional appearance of a nonzero toroidal structure.

\section{Summary and Discussion}

It was shown that with the toroidal pick-up coil array installed on ASDEX Upgrade an accurate toroidal mode number determination of modes is at least reliable up to $n \approx 16$. The determination of mode numbers at high frequencies is much improved by taking into account the intrinsic coil phases. In order to get good statistics during inter-ELM phases the method of ELM synchronization was used. Several magnetic activities with defined toroidal mode number could be identified during the ELM cycle at several radial positions or with different phase velocities. From the evolution of the mode numbers together with the evolution of the pedestal profiles at least six clearly separated phases during the cycle could be identified. These are four phases after the ELM crash, the pre-ELM phase and the ELM crash phase itself. During the first phase after the ELM crash no electromagnetic signal beside that from a core mode is visible. In all other phases around the ELM several branches with a variety of mode numbers with $n \leq 10$ but fixed $\mathrm{f} / \mathrm{n}$ values are present. This indicates that MHD modes with complex mode structure appear at distinct radial positions. From a comparison to the edge plasma rotation these modes could be located in the strong gradient region close to the plasma boundary. An exact determination of the position in terms of $q$ is not possible due to the unknown phase velocity and the large uncertainties of the plasma rotation velocity close to the separatrix.

Determination of mode numbers during the ELM crash is difficult because of the very fast development, but a low frequency that has been detected during the ELM fits to the drop of poloidal velocity in this phase and a continuous $\mathrm{n}$ structure around $n=3$ in this phase looks very similar to the dominant structure in the pre-ELM phase. Therefore, the ELM crash could be interpreted as the breakdown of the plasma rotation combined with the existence of a strong low $n$ mode structure. It remains unexplained what the origin of the $n=0$ and $n=-6$ structure that starts during the ELM phase and ends during the recovery phase is, but it might be connected to the appearance of an $n=0$ GAM or filamentary structures. 
Although ELM synchronization displays common features with good statistics the results for mode numbers in different phases also need to be validated with single ELM evaluations as the ELMs are always similar but still individual. Therefore, a single ELM mode number determination was performed for the phase just before the ELM crash showing, similar to the ELM synchronized picture, that dominant mode numbers jump from high to low $\mathrm{n}$ just before the crash, which is in good agreement with results from non-linear MHD code JOREK $[21,22]$.

Further work is necessary to place the here presented findings in a more general concept of the ELM cycle. Therefore, further comparisons to mode number results from JOREK are planned together with an analysis of a broader parameter space in terms of the edge current density and pressure gradients $\mathrm{j}$ and $\alpha$. For a better localization of the interELM modes also a poloidal mode number analysis is foreseen, although difficult, because of the need of a precise equilibrium close to the ELM crash.

\section{Acknowledgement}

This work has been carried out within the framework of the EUROfusion Consortium and has received funding from the Euratom research and training programme 2014-2018 under grant agreement No 633053. The views and opinions expressed herein do not necessarily reflect those of the European Commission.

F. M. Laggner is a fellow of the Friedrich Schiedel Foundation for Energy Technology.

[1] Zohm H 1996 Plasma Physics and Controlled Fusion 38 105-128 ISSN 0741-3335 URL http: //stacks. iop.org/0741-3335/38/i=2/a=001

[2] Connor J, Hastie R, Wilson H and Miller R 1998 Physics of plasmas 5 2687-2700 ISSN 1070-664X URL http://scitation.aip.org/content/aip/journal/pop/5/7/10.1063/1.872956

[3] Herrmann A, Eich T, Jachmich S, Laux M and Andrew P 2003 Journal of nuclear materials 313 759-767 ISSN 0022-3115 URL http://dx.doi.org/10.1016/S0022-3115(02)01422-8

[4] Perez C, Koslowski H, Hender T, Smeulders P and Loarte A 2004 Plasma Physics and Controlled Fusion 46 61-87 ISSN 0741-3335 URL http://stacks. iop.org/0741-3335/46/i=1/a=005

[5] Poli F M, Sharapov S E and Chapman S C 2008 Plasma Physics and Controlled Fusion 50095009 ISSN 0741-3335 URL http://stacks.iop.org/0741-3335/50/i=9/a=095009

[6] Diallo A, Groebner R J, Rhodes T L, Battaglia D J, Smith D R, Osborne T H, Canik J M, Guttenfelder W and Snyder P B 2015 Physics of Plasmas 22056111 URL http://scitation. aip.org/content/aip/journal/pop/22/5/10.1063/1.4921148

[7] Horváth L, Poloskei P Z, Papp G, Maraschek M, Schuhbeck K H, Pokol G I, the EUROfusion MST1 Team and the ASDEX Upgrade Team 2015 Plasma Physics and Controlled Fusion 57 125005 URL http://stacks.iop.org/0741-3335/57/i=12/a=125005

[8] Sommer F, Guenter S, Kallenbach A, Maraschek M and Boom J 2011 Plasma Physics and Controlled Fusion 53 085012 ISSN 0741-3335 URL http://stacks.iop.org/0741-3335/53/ $\mathrm{i}=8 / \mathrm{a}=085012$

[9] Winsor N, Johnson J and Dawson J 1968 Physics of fluids 11 2448-2450 ISSN 1070-6631 URL http://scitation.aip.org/content/aip/journal/pof1/11/11/10.1063/1.1691835

[10] Beurskens M, Arnoux G, Brezinsek A, Challis C, de Vries P, Giroud C, Huber A, Jachmich S, McCormick K, Pitts R, Rimini F, Alfier A, de la Luna E, Fundamenski W, Gerasimov 
S, Giovannozzi E, Joffrin E, Kempenaars M, Litaudon X, Loarer T, Lomas P, Mailloux J, Pasqualotto R, Pericoli-Ridolfini V, Pugno R, Rachlew E, Saarelma S, Solano E, Walsh M, Zabeo L, Zastrow K D and Contributors J E 2008 Nuclear Fusion 48095004 URL http: //stacks.iop.org/0029-5515/48/i=9/a=095004

[11] Dunne M, McCarthy P, Wolfrum E, Fischer R, Giannone L, Burckhart A and the ASDEX Upgrade Team 2012 Nuclear Fusion 52123014 URL http://stacks.iop.org/0029-5515/52/ $i=12 / a=123014$

[12] Fischer R, Fuchs C J, Kurzan B, Suttrop W and Wolfrum E 2010 Fusion science and technology 58 675-684 ISSN 1536-1055 URL http://dx.doi.org/10.13182/FST10-110

[13] Hatch D R, Told D, Jenko F, Doerk H and Dunne M G 2015 Nuclear fusion 55063028 ISSN 0029-5515 URL http://stacks.iop.org/0029-5515/55/i=6/a=063028

[14] Laggner F M, Wolfrum E, Cavedon M, Mink F, Viezzer E, Dunne M G, Manz P, Doerk H, Birkenmeier G, Fischer R, Fietz S, Maraschek M, Willensdorfer M, Aumayr F, the EUROfusion MST1 Team and the ASDEX Upgrade Team 2016 Plasma Physics and Controlled Fusion 58 065005 URL http://stacks.iop.org/0741-3335/58/i=6/a=065005

[15] Burckhart A, Wolfrum E, Fischer R, Lackner K and Zohm H 2010 Plasma Physics and Controlled Fusion 52105010 ISSN 0741-3335 URL http://stacks.iop.org/0741-3335/52/i=10/a= 105010

[16] Manz P, Boom J E, Wolfrum E and Birkenmeier G 2014 Plasma Physics and Controlled Fusion 56 035010 ISSN 0741-3335 URL http://stacks. iop.org/0741-3335/56/i=3/a=035010

[17] Pütterich T, Wolfrum E, Dux R and Maggi C F (ASDEX Upgrade Team) 2009 Phys. Rev. Lett. 102(2) 025001 URL http://link.aps.org/doi/10.1103/PhysRevLett.102.025001

[18] Viezzer E, Puetterich T, Conway G D, Dux R and Happel T 2013 Nuclear fusion 53053005 ISSN 0029-5515 URL http://stacks.iop.org/0029-5515/53/i=5/a=053005

[19] Coenen J W, Schweer B, Clever M, Freutel S and Schmitz O 2010 Journal of physics. B, Atomic, molecular and optical physics 43144015 ISSN 0953-4075 URL http://stacks.iop. org $/ 0953-4075 / 43 / i=14 / a=144015$

[20] Viezzer E, Pütterich T, Angioni C, Bergmann A, Dux R, Fable E, McDermott R, Stroth U, Wolfrum E and the ASDEX Upgrade Team 2014 Nuclear Fusion 54012003 URL http: //stacks. iop.org/0029-5515/54/i=1/a=012003

[21] Hoelz M, Guenter S and Wenninger R P 2012 Physics of plasmas 19082505 ISSN 1070-664X URL http://scitation.aip.org/content/aip/journal/pop/19/8/10.1063/1.4742994

[22] Krebs I, Hoelzl M, Lackner K and Guenter S 2013 Physics of plasmas 20082506 ISSN 1070-664X URL http://scitation.aip.org/content/aip/journal/pop/20/8/10.1063/1.4817953

[23] Maszl C, Naulin V, Brix M, Versloot T and Schrittwieser R 2011 Journal of Nuclear Materials 415 S451 - S454 ISSN 0022-3115 proceedings of the 19th International Conference on Plasma-Surface Interactions in Controlled Fusion URL http://www.sciencedirect.com/science/article/ $\mathrm{pii} / \mathrm{S} 0022311510010810$

[24] Kirk A, Muller H W, Herrmann A, Kocan M and Rohde V 2011 Plasma Physics and Controlled Fusion 53035003 ISSN 0741-3335 URL http://stacks.iop.org/0741-3335/53/ $i=3 / a=035003$

[25] Manz P, Carralero D, Birkenmeier G, Mller H W, Mller S H, Fuchert G, Scott B D and Stroth U 2013 Physics of Plasmas (1994-present) 20102307 URL http://scitation.aip.org/content/ aip/journal/pop/20/10/10.1063/1.4824799 